\title{
2020 KSC/KATRD Guideline for the Diagnosis and Treatment of Pulmonary Hypertension: Executive Summary
}

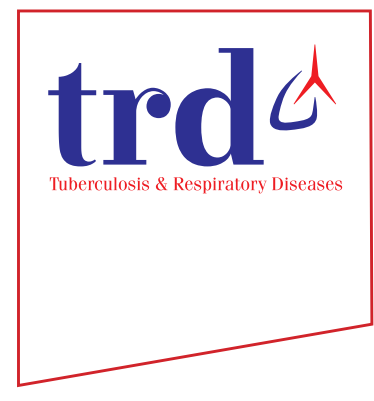

\author{
Jae-Hyeong Park, M.D., Ph.D. ${ }^{1}{ }^{(1)}$, Jin Oh Na, M.D., Ph.D. ${ }^{2}$, Jae Seung Lee, M.D., Ph.D. ${ }^{3}$, Yee Hyung \\ Kim, M.D., Ph.D. ${ }^{4}$, Hyuk-Jae Chang, M.D., Ph.D. ${ }^{5}{ }^{(}$, on behalf of Joint Task Force for the Diagnosis \\ and Treatment of Pulmonary Hypertension of the Korean Society of Cardiology (KSC) and the \\ Korean Academy of Tuberculosis and Respiratory Diseases (KATRD) \\ ${ }^{1}$ Department of Cardiology in Internal Medicine, Chungnam National University Hospital, Chungnam National University \\ College of Medicine, Daejeon, ${ }^{2}$ Cardiovascular Center, Korea University Guro Hospital, Korea University College of Medicine, \\ Seoul, ${ }^{3}$ Department of Pulmonary and Critical Care Medicine, Asan Medical Center, University of Ulsan College of Medicine, \\ Seoul, ${ }^{4}$ Division of Pulmonary and Critical Care Medicine, Department of Internal Medicine, Kyung Hee University Hospital \\ at Gangdong, Kyung Hee University School of Medicine, Seoul, ${ }^{5}$ Division of Cardiology, Severance Cardiovascular Hospital, \\ Yonsei University College of Medicine, Yonsei University Health System, Seoul, Republic of Korea
}

Pulmonary hypertension (PH) is a condition of increased blood pressure in the pulmonary arteries and is diagnosed with an increased a mean pulmonary artery pressure $\geq 25 \mathrm{~mm}$ Hg. This condition may be associated with multiple clinical situations. Based on pathophysiological mechanisms, clinical presentation, hemodynamic profiles, and treatment strategies, the patients were classified into five clinical groups. Although there have been major advances in the management of $\mathrm{PH}$, it is still associated with significant morbidity and mortality. The diagnosis and treatment of $\mathrm{PH}$ have been performed mainly by following European guidelines, even in Korea because the country lacks localized PH guidelines. European treatment guidelines do not reflect the actual status of Korea. Therefore, the European diagnosis and treatment of PH have not been tailored well to suit the needs of Korean patients with PH. To address this issue, we developed this guideline to facilitate the diagnosis and treatment of PH appropriately in Korea, a country where the consensus for the diagnosis and treatment of PH remains insufficient. This is the first edition of the guidelines for the diagnosis and treatment of PH in Korea, and it is primarily based on the '2015 ESC/ERS Guidelines for the diagnosis and treatment of pulmonary hypertension.' with the acceptance and adaptation of recent publications of PH.

Keywords: Pulmonary Hypertension; Treatment; Pulmonary Arterial Hypertension; Guidelines

Address for correspondence: Hyuk-Jae Chang, M.D., Ph.D.

Division of Cardiology, Severance Cardiovascular Hospital, Yonsei University College of Medicine, Yonsei University Health System, 50-1 Yonsei-ro, Seodaemun-gu, Seoul 03722, Republic of Korea

Phone: 82-2-2228-8454, Fax: 82-2-2227-8306, E-mail: hjchang@yuhs.ac.

Received: Feb. 5, 2021, Revised: May. 9, 2021, Accepted: Jun. 2, 2021, Published online: Jun. 4, 2021

@(c) it is identical to the Creative Commons Attribution Non-Commercial License (http://creativecommons.org/licenses/by-nc/4.0/) 


\section{Introduction}

Guidelines are established to help medical staff select the best approach for individual patients: the medical staff considers whether the impact of clinical outcomes and the severity of the effects outweigh the risks of specific diagnoses or treatment methods. Several international and domestic academic societies have provided many guidelines for treating various diseases. However, the diagnosis and the treatment of pulmonary hypertension $(\mathrm{PH})$ has mainly been performed by following European guidelines in Korea because the country lacks domestic guidelines. However, European treatment guidelines do not reflect the actual status of Korean patients. Therefore, new strategies of diagnosis and treatment should be fully developed for PH patients in Korea. Thus, we developed this guideline to help diagnose and treat PH appropriately in Korea, which is a country where the basis for the diagnosis and treatment of $\mathrm{PH}$ remains insufficient. The basic purpose of treatment guidelines is to assist medical staff in decision-making process through appropriate communication between physicians and patients in clinical practice. Our guideline is included in the first edition of guidelines for the diagnosis and treatment of PH in Korea. This edition is based on the '2015 ESC/ERS Guidelines for the diagnosis and treatment of pulmonary hypertension' with the acceptance and adaptation of recent publications of $\mathrm{PH}$. Moreover, we tried to include in this edition as many articles as possible on Korean data ${ }^{1}$.

The joint task force for the diagnosis and treatment of $\mathrm{PH}$ included the authors wrote and reviewed panels without conflict of interest. The panel of writers, which included five members of the Korean Society of Cardiology (KSC) and the Korean Academy of Tuberculosis and Respiratory Diseases (KATRD), created a draft that included an 'up-to-date' published evidence for the diagnosis, treatment, prevention, and rehabilitation of $\mathrm{PH}$ patients, including those living in Korea. The review panel comprised of 15 members, and they represented the opinions of 11 associated academic societies, including the Korean College of Rheumatology (KCR), the Korean Heart Rhythm Society (KHRS), the Korean Pediatric Heart Society (KPHS), the Korean Society of Heart Failure (KSHF), the Korean Society of Cardiovascular Imaging (KOSCI), the Korean Society of Echocardiography (KSE), the Korean Society of Interventional Cardiology (KSIC), the Korean Society for Transplantation (KST), the Korean Society for Laboratory Medicine (KSLM), the Korean Pulmonary Hypertension Society in Korean Society of Hypertension (KPHS/KSH), and the Korean Association of Clinical Cardiology (KACC). To avoid conflicts of interests, the task force that prepared guidelines for PH did not seek any financial support from related industries.

For the accommodation and adaptation of this guideline, we searched a total of 31,077 articles that were published across a 10-year period. These articles were obtained from the following three databases: MEDLINE, PubMed, and EMBASE. After evaluating these articles with K-AGREE II (Appraisal of Guideline for Research and Evaluation II), including six domains and 23 items, we finally selected the ' 2015 ESC/ERS Guidelines for the diagnosis and treatment of pulmonary hypertension' as a reference standard. Then, we partially adapted the 'Guidelines for the Treatment of Pulmonary Hypertension (Japanese Circulation Society 2017/Japanese Pulmonary Circulation and Pulmonary Hypertension Society 2017) ${ }^{1,2}$.

Table 1. Strength of recommendations

\begin{tabular}{|cll|}
\hline Criterion & \multicolumn{1}{c|}{ Definition } & \multicolumn{1}{c|}{ Suggestion } \\
\hline Class I & $\begin{array}{l}\text { Benefit }>>\text { risk } \\
\text { Procedure or treatment is beneficial, useful, and effective. }\end{array}$ & $\begin{array}{l}\text { Procedure/treatment should be performed. } \\
\text { Is recommended/is indicated }\end{array}$ \\
Class IIa & $\begin{array}{l}\text { Benefit>risk } \\
\text { Evidence or opinion is useful or efficacious. }\end{array}$ & $\begin{array}{l}\text { It is reasonable to perform this procedure/treatment. } \\
\text { Should be considered }\end{array}$ \\
Class IIb & $\begin{array}{l}\text { Benefit } \geq \text { risk } \\
\text { Additional studies should be conducted to address broad objectives } \\
\text { because usefulness/efficacy is well established to a lesser extent. }\end{array}$ & $\begin{array}{l}\text { Procedure/treatment may be considered. } \\
\text { Class III }\end{array}$ \\
& $\begin{array}{l}\text { Benefit } \leq \text { risk } \\
\text { Procedure or treatment is not useful or is potentially harmful }\end{array}$ & $\begin{array}{l}\text { Procedure/treatment should not be performed. } \\
\text { Is not recommended }\end{array}$ \\
\hline
\end{tabular}

Table 2. Level of evidence

\begin{tabular}{|cl|}
\hline Level & Definition \\
\hline A & Multiple populations are evaluated in multiple randomized clinical trials or meta-analyses. \\
B & Limited populations are evaluated in a single randomized trial or in large non-randomized studies. \\
C & $\begin{array}{c}\text { A consensus on the opinion of experts: very limited populations were evaluated in small studies, retrospective studies, or in } \\
\text { registries }\end{array}$ \\
\hline
\end{tabular}


Thereafter, we reviewed consensus manuscripts and included them in this process. As shown in Tables 1 and 2, the level of evidence and the strength of recommendations for each item were graded and presented according to a predefined scale.

This guideline represents the official information on $\mathrm{PH}$, which was presented by selected members of the KSC, KATRD, KPHS, and KPHS/KSH, and the guideline will be updated periodically. The full version of the $\mathrm{PH}$ guideline can be found at the official websites of the following academic societies: the KSC (http://www.circulation.or.kr), the KATRD (http:// www.lungkorea.org), and the Korean Medical Guideline Information Center (https://www.guideline.or.kr/evaluation/index. php?sub=1). In this guideline, the recommendations mentioned were prepared to help medical experts make decisions in their daily work. The existing evidence of individual issues was evaluated and summarized in this guideline, but the final treatment decision for each patient had to be made by an attending physician. After carefully discussing with the patients and their caregivers. In addition, it is the responsibility of the individual medical practitioner to check related regulations such as various permits and insurance benefits that change concerning medications and devices when prescribing.

In the future, follow-up investigations should be conducted to verify whether the actual daily practice is performed in accordance with the recommendations of these PH guidelines. Apart from this process, it is necessary to create a series of virtuous cycles, including clinical research, guideline preparation, the dissemination, and the clinical application of these guidelines.

\section{Definition and Classification of $\mathrm{PH}$}

1. To confirm the diagnosis of $\mathrm{PH}$ and to support treatment decisions, medical staff should perform right heart catheterization (RHC) only after ensuring that the patient is in resting position (The strength of recommendation I, The level of evi- dence $C$ ).

2. It is recommended that medical staff performs RHC at expert centers (The strength of recommendation I, The level of evidence B).

3. Vasoreactivity testing is recommended in patients with any of the following conditions: idiopathic pulmonary arterial hypertension (PAH) (IPAH), hereditary PAH (HPAH), and $\mathrm{PAH}$. These conditions may be induced by drugs or toxins to help us detect patients who experienced long-term benefits with high doses of a calcium channel blocker (CCB, The strength of recommendation I, The level of evidence $C$ ).

\section{Definition of PH}

In the resting position, the normal value of mean pulmonary arterial pressure (mPAP) is $14 \pm 3 \mathrm{~mm} \mathrm{Hg}$; the upper limit of normal value is about $20 \mathrm{~mm} \mathrm{Hg}$, which is twice the standard deviation while assuming a normal distribution of pulmonary arterial pressure $(\mathrm{PAP})^{3}$. Thus, $\mathrm{PH}$ is defined as an increase in $\mathrm{mPAP}$ to $\geq 25 \mathrm{~mm} \mathrm{Hg}$ in the resting position. Moreover, mPAP should be determined by performing $\mathrm{RHC}^{1,4}$.

Recently, the 6th World Symposia on Pulmonary Hypertension (WSPH) took place in Nice, France. The task forces presented their consensus opinion that $\mathrm{PH}$ should be defined as $\mathrm{mPAP}>20 \mathrm{~mm} \mathrm{Hg}$ rather than $\mathrm{mPAP} \geq 25 \mathrm{~mm} \mathrm{Hg}$ because two standard deviations were above the normal mPAP value. Thus, $\mathrm{PH}$ is the threshold for abnormal PAP ${ }^{5}$. After holding an active debate over why the criteria for $\mathrm{PH}$ was changed to $\mathrm{mPAP}>20$ $\mathrm{mm} \mathrm{Hg}$ in this guideline, we retained the threshold of $\mathrm{PH}$ as $\mathrm{mPAP} \geq 25 \mathrm{~mm} \mathrm{Hg}$. This is because additional research studies should be conduct to support these new criteria and the consensus of medical experts.

PH can be defined hemodynamically by using various combinations of following parameters: PAP, pulmonary artery wedge pressure, cardiac output (CO), diastolic pressure gradient, and pulmonary vascular resistance (PVR). Table 3 describes the different hemodynamic classifications of $\mathrm{PH}$.

Table 3. Hemodynamic definition of PH

\begin{tabular}{|lll|}
\hline \multicolumn{1}{|c|}{ Definition } & \multicolumn{1}{c|}{ Characteristic } & \multicolumn{1}{c|}{ Clinical PH group } \\
\hline Pre-capillary PH & $\mathrm{mPAP} \geq 25 \mathrm{~mm} \mathrm{Hg}$ & $1,3,4,5$ \\
& $\mathrm{PAWP} \leq 15 \mathrm{~mm} \mathrm{Hg}$ & 2,5 \\
Post-capillary PH & $\mathrm{mPAP} \geq 25 \mathrm{~mm} \mathrm{Hg}$ \\
& $\mathrm{PAWP}>15 \mathrm{~mm} \mathrm{Hg}$ \\
\hline Isolated post-capillary PH & + & \\
& $\mathrm{DPG}^{*}<7 \mathrm{~mm} \mathrm{Hg}$ and/or PVR $\leq 3 \mathrm{WU}^{\dagger}$ \\
Combined post-capillary and pre-capillary PH $(\mathrm{Cpc}-\mathrm{PH})$ & + \\
& $\mathrm{DPG} \geq 7 \mathrm{~mm} \mathrm{Hg}$ and/or PVR $>3 \mathrm{WU}$ \\
\hline
\end{tabular}

Modified from Galie N et al. Eur Heart J 2016;37:67-119, with permission from Oxford University Press ${ }^{1}$.

${ }^{*} \mathrm{DPG}=$ diastolic PAP-mean PAWP. ${ }^{\dagger} \mathrm{WU}$ are preferred to dynes $\mathrm{s} / \mathrm{cm}^{5}$, and $1 \mathrm{WU}$ is about 80 dynes $\cdot \mathrm{s} / \mathrm{cm}^{5}$.

PH: pulmonary hypertension; mPAP: mean pulmonary artery pressure; PAWP: pulmonary artery wedge pressure; DPG: diastolic pressure gradient; PVR: pulmonary vascular resistance; WU: Wood units. 
RHC should be performed to define $\mathrm{PH}$ and to assess the severity of $\mathrm{PH}$ and to check the acute vasoreactivity in expert centers. When RHC is performed in expert centers, both the complication rate and the mortality rate are very low at $1.1 \%$ and $0.06 \%$, respectively ${ }^{6}$. Vasoreactivity testing is performed in patients with any of the following conditions: IPAH, HPAH, and PAH, which could be induced by drugs or toxins to detect patients who can have long-term benefits from high-dose of a $\mathrm{CCB}^{7,8}$.

\section{Classifications of PH}

There are five clinical classifications of PH patients, with similar pathophysiological mechanisms, clinical presentation, hemodynamic profiles, and treatment strategies (Table 4$)^{1,9}$. In $\mathrm{PAH}$ patients with an acute vasodilator response, the survival rate was dramatically improved when they treated with longterm $\mathrm{CCB}^{7,8}$. Therefore, it is necessary to identify these patients where pulmonary vasoconstriction plays an important role in PAH pathophysiology is vital. Thus, these PAH patients with long-term responders to CCBs were incorporated into the group 1.5. The causes of pulmonary veno-occlusive disease (PVOD) and pulmonary capillary hemangiomatosis (PCH) are similar, and these conditions are closely associated with PAH. Therefore, patients with PVOD or PCH were included in the group 1.6. Persistent pulmonary hypertension of the newborn which exhibits different patterns from conventional PAH, was classified into a separate disease category, which was included within the group 1.7 .

Both congenital and acquired heart diseases causing postcapillary PH were included in the group 2. Patients with PAH associated with congenital heart disease are classified into four groups according to their clinical profiles: (1) Eisenmenger's syndrome, (2) PAH associated with prevalent systemic and pulmonary shunts, (3) PAH with small/coincidental defects, and (4) PAH after defect correction ${ }^{1,10}$.

Group $3 \mathrm{PH}$ is due to lung diseases and/or hypoxemia. In this group, the common causes of group $3 \mathrm{PH}$ are interstitial lung disease and chronic obstructive pulmonary disease (COPD). Severe PH is usually uncommon in patients with severe interstitial lung disease or COPD. However, severe PH

Table 4. Clinical classification of pulmonary hypertension

Group 1. Pulmonary arterial hypertension (PAH)

Group 2. Pulmonary hypertension (PH) due to left heart diseases

Group 3. PH due to lung diseases and/or hypoxia

Group 4. Chronic thromboembolic pulmonary hypertension and other pulmonary artery obstructions

Group 5. PH with unclear and/or multifactorial mechanisms

Modified from Galie N et al. Eur Heart J 2016;37:67-119, with permission from Oxford University Press ${ }^{1}$. can be associated with the combined emphysema/fibrosis syndrome.

Group $4 \mathrm{PH}$ was changed to include chronic thromboembolic pulmonary hypertension (CTEPH), and other pulmonary artery obstructions, including pulmonary sarcoma or angiosarcoma, other intravascular tumors, arteritis without connective tissue disease, congenital pulmonary artery stenosis, and parasites ${ }^{10}$.

Chronic hemolytic anemia is associated with an increased risk of PH. Pre-capillary PH associated with chronic hemolytic anemia is different from other types of PAH in terms of pathological characteristics (plexiform lesions), hemodynamic characteristics (low PVR and increased CO), and response to specific treatments for PAH (no response). This group was considered a different clinical condition, and the classification was changed from group 1 to group 5 .

\section{Epidemiology and Genetics of PAH}

\section{Epidemiology of PAH}

The data pertaining to the global incidence of $\mathrm{PH}$ is insufficient. The prevalence of PAH in Europe is in the range 15-60 patients per million adult population, and the incidence of PAH in Europe is $5-10$ cases per million adult population per year ${ }^{1,11}$.

The epidemiologic data of $\mathrm{PH}$ in Korea is also scant. A retrospective study was based on the data collected from the Health Insurance Review and Assessment Service (HIRA) claim database (from 2008 to 2016): there were 1,307 newly diagnosed PAH patients in total. The mean age of diagnosis was $44 \pm 13$ years. Among them, $69.3 \%$ of the patients were female, and $30.7 \%$ of the patients were male ${ }^{12}$. The incidence of PAH in Korea was 4.8 patients per million adult population per year. In total, $625 \mathrm{PAH}$ patients were enrolled in the Korean Registry of Pulmonary Arterial Hypertension (KORPAH) study, which is the first prospective registry that included participants from four domestic societies (KSC, KCR, KPHS, and KATRD $)^{13}$. The mean age of participants was $48 \pm 16$ years. Among them, 503 (80.5\%) of the patients were females. The incidence of PAH was 1.9 per million adult population per year $^{13}$. This data showed that a low proportion of PAH patients who are diagnosed and treated remains low, indicating that a more-active patient screening programs should be more actively implemented in Korea. However, clinicians should interpret these data with caution because the frequency of RHC performance was very low (39.8\%), and the degree of participation varied according to each academic society. Globally, systemic sclerosis is the most common cause of PAH. In Korea, it seems that many PAH patients have an associated complication of systemic lupus erythematosus.

The clinical pattern and the incidence of PAH changed ac- 
cording to the clinical situation of patients ${ }^{14}$. In the U.S. National Institutes of Health registry, which was first implemented in 1981, the mean age of patients with IPAH was 36 years. After including more PAH patients, the average age of patients is currently 50-65 years in this registry. The overall prognosis seems to have improved over a period of time.

According to HIRA database, the average age of group 1 PAH patients was $44 \pm 13$ years, and the mean age of IPAH patients was $48 \pm 13$ years in Korea ${ }^{12}$. The average age of PAH patients in the KORPAH registry was $48 \pm 16$ years, and the average age of IPAH patients was 45 years ${ }^{13}$.

\section{Genetics of PAH}

Mutations of bone morphogenic receptor protein 2 (BMPR2) are the most well-known genetic anomalies, and heterogynous BMPR2 mutations account for about $75 \%$ of familial PAH patients and approximately $25 \%$ of sporadic $\mathrm{PAH}$ patients $^{15}$. The BMPR2 gene encodes a type 2 receptor of bone morphogenetic proteins, which control vascular cell proliferation. In the PILGRIM study, which examined the genetic mutation and the clinical features of BMPR2 were examined in 73 patients of Korea. The results indicated that $22 \%$ of patients with IPAH had mutations in BMPR2 gene ${ }^{16}$. The patients with BMPR2 mutations were relatively younger (27 years vs. 47 years, $\mathrm{p}=0.02)$ and had a higher mPAP than non-carriers (64 $\mathrm{mm} \mathrm{Hg}$ vs. $51 \mathrm{~mm} \mathrm{Hg}$, $\mathrm{p}<0.05$ ).

The heritable conditions of PVOD/PCH were caused by biallelic mutations of eukaryotic translation initiation factor 2 alpha kinase 4 (EIF2AK4), which encodes a serine-threonine kinase in all the eukaryotes ${ }^{17}$.

\section{Diagnosis of $\mathrm{PH}$}

1. Transthoracic echocardiography is a first-line noninvasive diagnostic test in clinically suspected $\mathrm{PH}$ cases (The strength of recommendation I, The level of evidence $C$ ).

2. Ventilation/perfusion lung scan should be performed in cases with unexplained PH to exclude CTEPH (The strength of recommendation $I$, The level of evidence $C$ ).

3. Computerized tomography (CT) pulmonary angiography is recommended to evaluate patients with CTEPH (The strength of recommendation I, The level of evidence $C$ ).

4. Biochemistry, hematology, immunology, human immunodeficiency virus (HIV) tests, and thyroid function tests are recommended to evaluate the specific associated condition of all the patients with PAH (The strength of recommendation I, The level of evidence $C$ ).

\section{Diagnostic tests of PH}

The diagnosis of PH was conducted in cases with a clinical suspicion of the disease; the symptoms or findings of physical examination caused clinical suspicion of PH. Although RHC is performed to determine hemodynamic criteria, it is also mandatory in the diagnosis of PH. Nevertheless, several diagnostic tests is necessary. Apart from determining the cause of $\mathrm{PH}$, it is also essential to evaluate the severity of the disease in a $\mathrm{PH}$ patient. The diagnosis has to be performed systematically and consistently. To perform a comprehensive evaluation of the patient, physicians determined following parameters: functional classification, exercise capacity, echocardiographic findings, blood tests, and hemodynamic status ${ }^{18}$.

Transthoracic echocardiography is a first-line, non-invasive diagnostic test, which is performed on clinically suspected cases of PH. The probability of PH can be assessed by performing transthoracic echocardiography, which is a test that evaluates the effects of PH and estimates PAP with Doppler measurements ${ }^{1,19}$. When $\mathrm{PH}$ is probable, further evaluation tests such as $\mathrm{RHC}$ are recommended to confirm the diagnosis of PH.

In ventilation/perfusion lung scan, a normal or low probability result effectively excludes the possibility of CTEPH. Therefore, this test should be performed to evaluate CTEPH in patients with unexplained $\mathrm{PH}$.

CT imaging can provide more important information on pulmonary vascular structure, and parenchymal and mediastinal abnormalities; these conditions may suggest the development of PH. To evaluate patients with CTEPH, we performed CT pulmonary angiography.

The biochemistry, hematology, immunology, HIV diagnosis tests, and thyroid function tests are recommended to evaluate the specifically associated condition of all patients with PAH.

\section{Diagnostic algorithm}

Figure 1 describes the diagnostic algorithm of $\mathrm{PH}$. The suspected cases of PH were characterized by symptoms, signs, or clinical history. These patients had to undergo echocardiographic examination to determine the probability of $\mathrm{PH}$. The more common clinical groups of PH (group 2 [PH due to left heart disease] and group 3 [PH due to lung disease and/ or hypoxia]) had to be initially identified from patients with echocardiographic findings, which were compatible with $\mathrm{PH}$. Then, group 4 (CTEPH) was distinguished by performing ventilation/perfusion lung scan on $\mathrm{PH}$ patients, which did not show any evidence of group 2 or group $3 \mathrm{PH}$. Furthermore, $\mathrm{PH}$ patients that could not be included in group $4 \mathrm{PH}$ were classified by the presence of various causes of PAH (group 1) and rarer causes of $\mathrm{PH}$ (group 5). 


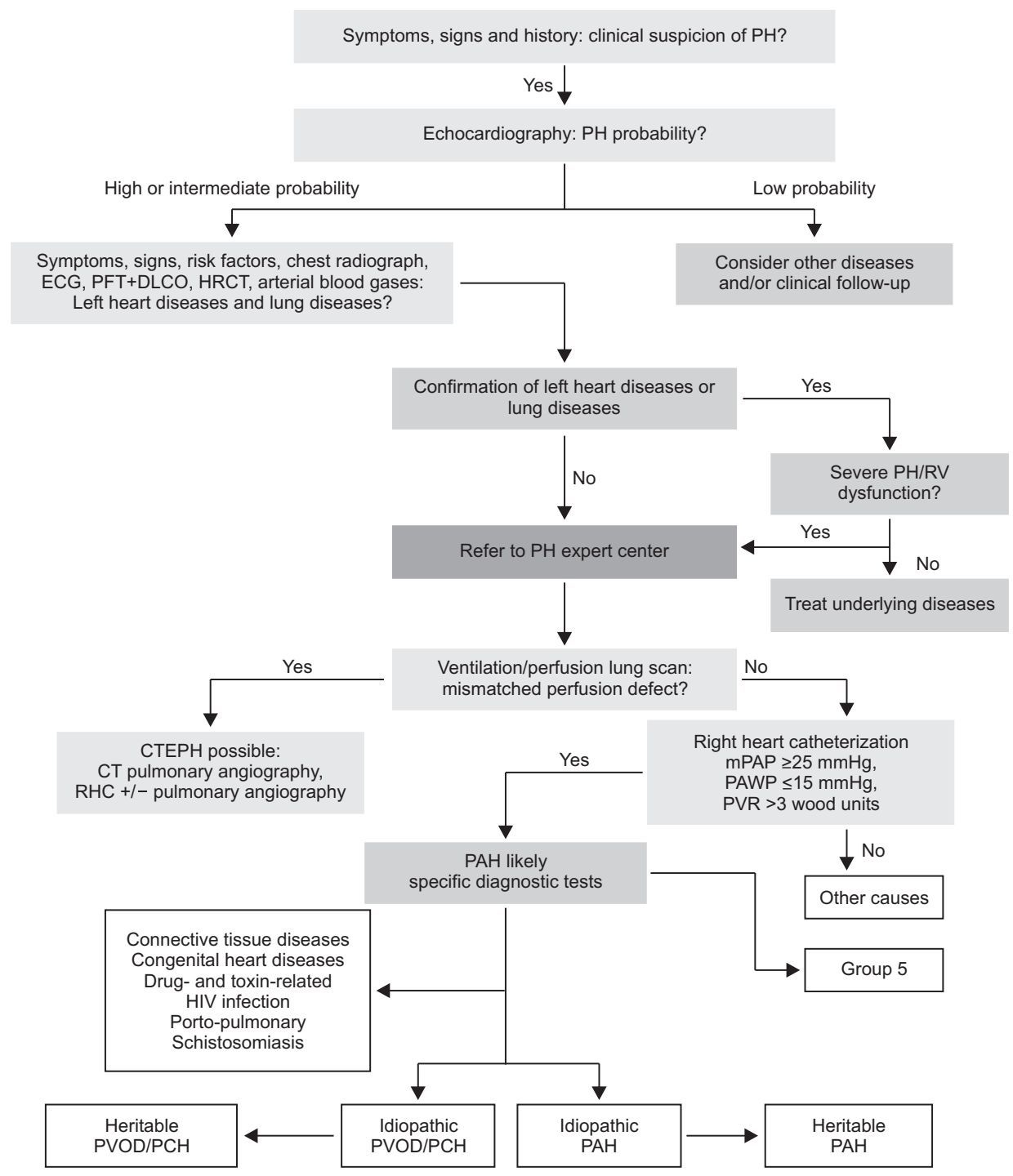

Figure 1. Diagnostic algorithm of pulmonary hypertension. Modified from Galie $\mathrm{N}$ et al. Eur Heart J 2016;37:67-119, with permission from Oxford University Press ${ }^{1}$. PH: pulmonary hypertension; ECG: electrocardiography; PFT: pulmonary function test; DLCO: diffusing capacity of the lung for CO; HRCT: high-resolution computed tomography; RV: right ventricular; CTEPH: chronic thromboembolic pulmonary hypertension; CT: computed tomography; RHC: right heart catheterization; mPAP: mean pulmonary artery pressure; PAWP: pulmonary artery wedge pressure; PVR: pulmonary vascular resistance; PAH: pulmonary arterial hypertension; HIV: human immunodeficiency virus; PVOD/PCH: pulmonary veno-occlusive disease or pulmonary capillary hemangiomathosis.

\section{Prognostic Evaluation and Risk Assessment of PAH}

During the clinical evaluation of patients with $\mathrm{PAH}$, it is necessary to provide valuable information pertaining to disease severity, improvement, deterioration, and stability. During follow-up visits, physicians note down basic history of patients, which includes changes in exercise capacity, episodes of chest pain, arrhythmia, hemoptysis or fainting, and changes in concomitant medications. In addition, physicians also evaluate whether the prescribed medications are compliant with the patient's condition. By performing physical examination, physicians gather information on the presence or absence of distal or central cyanosis, edema, ascites, pleural effusion, and the dilatation of jugular vein. Moreover, they also measure the heart rate, rhythm, and blood pressure of $\mathrm{PAH}$ patients.

Although there are considerable differences among clini- cians $^{20}$, the evaluation of dyspnea is performed according to the World Health Organization (WHO) functional class criterion as it is the most powerful indicator that predicts clinical prognosis during a follow-up visit and at the time of diagnosis.

The 6-minute walking test (6MWT), which evaluates the submaximal exercise capacity of a patient, is the most widely used exercise test at many PH centers. This test is easy to perform, inexpensive, and familiar to both the patients and the medical staff. Just like other PH assessments, physicians always interpret the results of 6MWT in clinical context. In general, the cardiopulmonary exercise test (CPET) is performed to evaluate maximal exercise capacity of $\mathrm{PH}$ patient. Thus, it provides important information on gas exchange, ventilatory efficiency, and cardiac function during the time of exercise, so it is used to evaluate the exercise capacity of a patient. It offers information on the end-tidal partial pressure of carbon dioxide $\left(\mathrm{PCO}_{2}\right)$, ventilatory equivalents of carbon dioxide $\left(\mathrm{VE} / \mathrm{VCO}_{2}\right)$, 
oxygen/pulse $\left(\mathrm{VO}_{2} / \mathrm{HR}\right)$, and peak oxygen uptake (peak $\left.\mathrm{VO}_{2}\right)$. It was found that $\mathrm{PAH}$ patients had low $\mathrm{PCO}_{2}$, high $\mathrm{VE} / \mathrm{VCO}_{2}$, low $\mathrm{VO}_{2} / \mathrm{HR}$, and low peak $\mathrm{VO}_{2}^{21}$.

Although various biochemical markers have been studied, physicians have not found a single specific indicator that reflects the degree of pulmonary vascular remodeling or the severity of PAH. Among these biomarkers, B-type natriuretic peptide (BNP) and N-terminal pro-BNP (NT-proBNP) concentrations correlate with myocardial dysfunction. Thus, they provide prognostic information both during the follow-up visits and at the time of diagnosis ${ }^{22}$. But, they are nonspecific biomarkers of PH and are associated with many cardiovascular diseases. Therefore, these levels of biomarkers should be interpreted in the clinical context.

Traditionally, risk assessment of PAH patients was performed in a multidimensional setting by performing imaging studies and by examining hemodynamic profiles. Thus, physicians consider the following parameters: symptoms, the WHO functional class, the clinical signs of right heart failure, the results of $6 \mathrm{MWT}$ or CPET, the expression of BNP/NTproBNP, the presence of pericardial effusion, and the size of right atrial area. Since these variables are partially overlapping, it is difficult to measure all the variables at each follow-up visit. Therefore, a simplified evaluation system was presented at the sixth WSPH. Since it facilitated risk assessment, we adapted and included it in our guideline (Table 5$)^{23}$. This is because it is unnecessary to evaluate all the parameters of PAH patients at every follow-up visit. However, clinicians should determine the functional class and check the patient's exercise capacity by conducting 6MWT or CPET. It is also recommended to measure the biomarkers, BNP/NT-proBNP. Moreover, clinicians should also obtain information on the right ventricular function by performing an echocardiographic examination of PAH patients.

However, in the risk assessment proposed above, most of the variables are based on the published data and expert opinion. Therefore, clinicians should exercise caution while applying these variables to individual patients.

\section{Treatment of $\mathrm{PH}$}

1. CCBs are recommended in patients with IPAH, HPAH and drug-induced PAH who have a positive vasoreactivity test (The strength of recommendation I, The level of evidence $C$ ).

2. Initial monotherapy or combination therapy of approved drugs should be provided to treatment-naïve patients with $\mathrm{PAH}$ and to patients with low- or intermediate-risk of PAH (The strength of recommendation I, The level of evidence $A$ ).

3. A sequential combination therapy of approved drugs is recommended in PAH patients who show an inadequate treatment response to initial monotherapy or initial double combination therapy (The strength of recommendation I, The level of evidence $B$ ).

4. The use of PAH-approved drugs is not recommended to $\mathrm{PH}$ patients with left heart disease or lung diseases (The strength of recommendation III, The level of evidence $C$ ).

5. Surgical pulmonary endarterectomy and deep hypothermic circulatory arrest is recommended for CTEPH patients with operability (The strength of recommendation I, The level of evidence $B$ ).

6. Riociguat is recommended in symptomatic CTEPH patients with persistent or recurrent lesions after surgical treatment or in inoperable CTEPH patients (The strength of

Table 5. Risk assessment in patients with pulmonary arterial hypertension

\begin{tabular}{|lccc|}
\hline \multicolumn{1}{|c}{ Criterion } & Low risk $^{*}$ & ${\text { Intermediate } \text { risk }^{\dagger}}^{\dagger}$ & ${\text { High } \text { risk }^{\dagger}}^{{ }^{*}}$ \\
\hline Estimated l-year mortality, \% & $<5$ & $5-10$ & $>10$ \\
\hline WHO functional class & I, II & III & IV \\
\hline 6-minute walking distance $(\mathrm{m})$ & $>440$ & $165-440$ & $<165$ \\
\hline Biochemical test & & & $>300$ \\
\hline BNP $(\mathrm{ng} / \mathrm{L})$ & $<50$ & $50-300$ & $>1,400$ \\
\hline NT-proBNP $(\mathrm{pg} / \mathrm{mL})$ & $<300$ & $300-1,400$ & \\
\hline Hemodynamic profile & & & $>14$ \\
\hline $\mathrm{RA}$ pressure $(\mathrm{mmHg})$ & $<8$ & $8-14$ & $<2.0$ \\
\hline $\mathrm{CI}\left(\mathrm{L} / \mathrm{min} / \mathrm{m}^{2}\right)$ & $\geq 2.5$ & $2.0-2.4$ & $<60$ \\
\hline $\mathrm{SvO}_{2}(\%)$ & $>65$ & $60-65$ & \\
\hline
\end{tabular}

Modified from Thomas CA et al. Pulm Ther 2020;6:9-22, according to the Creative Commons license ${ }^{23}$.

*At least three low risk criteria and no high-risk criterion. ${ }^{\dagger}$ Definitions of low or high risk are not fulfilled. ${ }^{*}$ At least two high-risk criteria, including $\mathrm{CI}$ or $\mathrm{SvO}_{2}$.

WHO: World Health Organization; BNP: B-type natriuretic peptide; NT-proBNP: N-terminal pro-B-type natriuretic peptide; RA: right atrial; CI: cardiac index; $\mathrm{SvO}_{2}$ : mixed venous oxygen saturation. 
recommendation I, The level of evidence $B$ ).

Initially, PAH patients should be managed with following general measures: physical activity, supervised rehabilitation, inhibition of pregnancy, birth control pills and post-menopausal hormonal therapy, prompt preprocedural management of elective surgery, prevention of infection, psychosocial support, adherence to treatments, genetic counseling, and recommended precautions during traveling. Supportive therapy for PAH patients includes oral anticoagulants, diuretics, oxygen, and digoxin.

Drug treatment for PAH patients should be provided on the basis of diagnosis, symptoms, and results of acute vasoreactivity test. Without contraindication to using CCBs, CCBs should be considered for PAH patients who show a favorable response to acute vasoreactivity test, which is performed along with RHC at the time of diagnosis. The choice of CCB is based on the heart rate at baseline: nifedipine (120-240 mg/ day) and diltiazem (240-720 mg/day) are the CCBs that are frequently prescribed to $\mathrm{PAH}$ patients ${ }^{7,8}$.

When PAH patients do not show vasoreactivity, clinicians can prescribe PAH-specific drugs according to the grade of recommendation and the level of evidence for treatment. However, it is not necessary to prescribe PAH-specific drugs to PAH patients who do not show any symptoms (WHO functional class I). A close follow-up is required to determine if and when symptoms occur in these patients and before deciding whether PAH-specific drugs should be prescribed ${ }^{18}$. For PAH patients in WHO functional class II or higher, the treatment is according to their individual indications. Figure 2 shows the treatment algorithm.

Clinicians prescribed the following PAH-specific drugs: endothelin receptor antagonists, phosphodiesterase type5 inhibitors (PDE-5I), guanylate cyclase stimulators (cGC), prostanoids, and IP-receptor agonists. An initial monotherapy or combination therapy of PAH-specific drugs was recommended to newly diagnosed PAH patients and to patients with low- or intermediate-risk of PAH but without vasoreactivity. An initial combination therapy included intravenous prostacyclin analogs, which is prescribed to $\mathrm{PAH}$ patients of WHO functional class IV. An intravenous epoprostenol is the first line of treatment because it has reduced the initial mortality of high-risk PAH patients. However, this drug is not available in Korea (as of October 2020). Therefore, it is necessary to actively implement other types of initial upfront combination therapy. After providing initial monotherapy or initial double combination therapy, the treatment response is inadequate in the reassessment period of 3-6 months, then clinicians have to administer a sequential double or triple combination therapy of approved drugs to symptomatic PAH patients. The combination of PDE-5I and cGC drugs shows contraindications.

Pulmonary endarterectomy (PEA) in deep hypothermic circulatory arrest is recommended for CTEPH patients with operability assess by a multidisciplinary team. Riociguat can

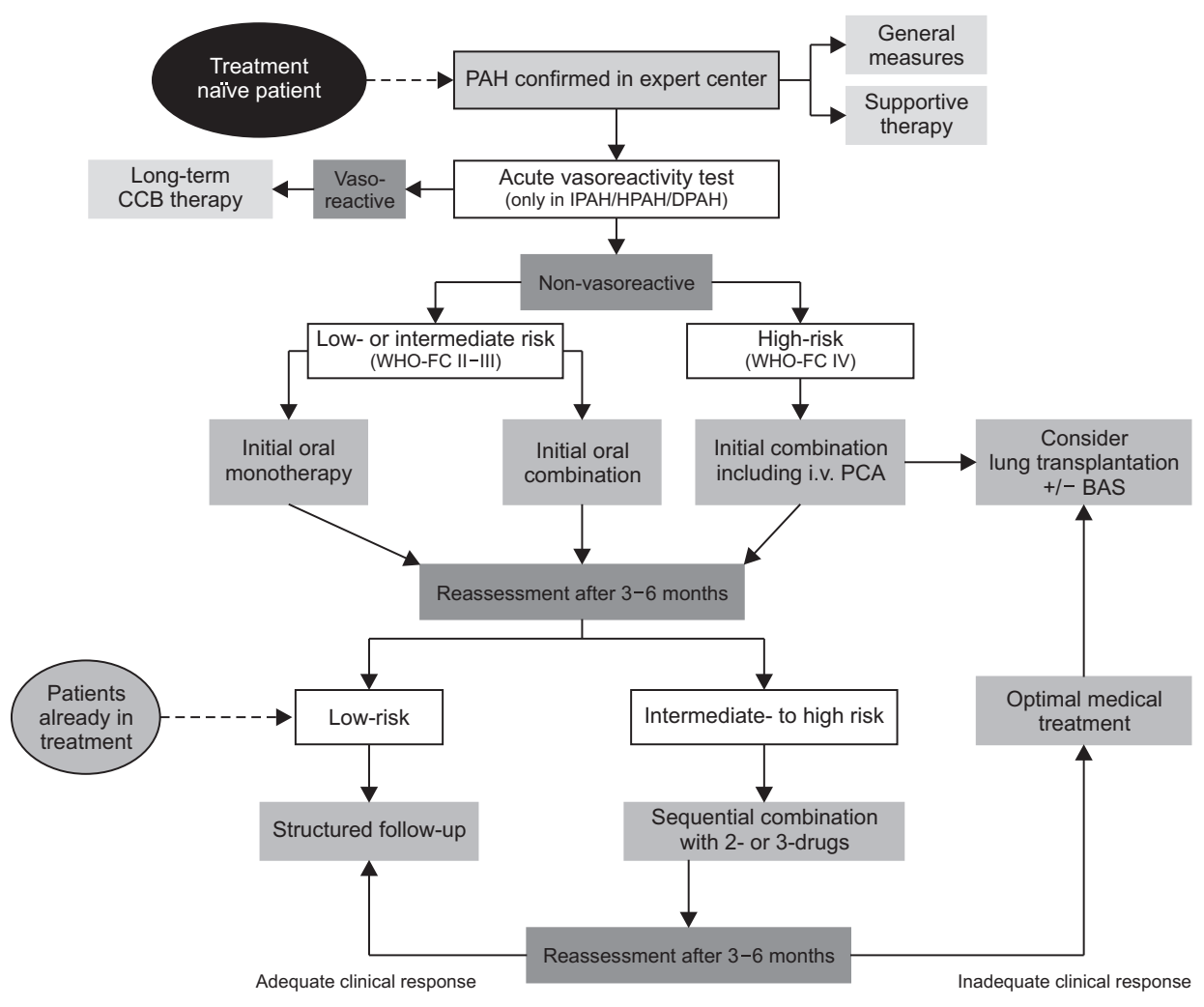

Figure 2. Treatment algorithm of pulmonary arterial hypertension. Modified from Thomas CA et al. Pulm Ther 2020;6:9-22, according to the Creative Commons license ${ }^{23}$. PAH: pulmonary arterial hypertension; CCB: calcium channel blocker; IPAH: idiopathic pulmonary arterial hypertension; HPAH: heritable pulmonary arterial hypertension; DPAH: drug- or toxin-induced pulmonary arterial hypertension; WHO-FC: World Health Organization functional class; PCA: prostacyclin analogue; BAS: balloon atrial septostomy. 
be prescribed to inoperable CTEPH patients or in patients with persistent or recurrent lesions after undergoing PEA. Moreover, balloon pulmonary artery angioplasty may be an intervention for inoperable CTEPH patients.

Even after providing initial monotherapy or combination therapy to PAH patients, the clinical response may be inadequate. In such a situation, $\mathrm{PAH}$ patients must be further examined to determine whether they are suitable candidates for lung transplantation.

\section{Conclusion}

Over the past decades, there have been major advances in the management of PH. However, the morbidity and mortality of PH is still significant. Moreover, the strategy used for the diagnosis and treatment of $\mathrm{PH}$ is mainly associated with European guidelines, even in Korea. This is because the country lacks localized guidelines for the diagnosis and treatment of PH. Since European treatment guidelines do not reflect the actual status of Korean patients, the diagnosis and treatment have not been effective on Korean patients with PH. Therefore, we developed a new guideline to help diagnose and treat PH cases appropriately in Korea, which is a country with insufficient data for the diagnosis and treatment of $\mathrm{PH}$.

\section{Authors' Contributions}

Conceptualization: Park JH, Oh JO, Lee JS, Kim YH, Chang HJ. Data curation: Park JH, Chang HJ. Writing - original draft preparation: Park JH, Chang HJ. Writing - review and editing: Park JH, Oh JO, Lee JS, Kim YH, Chang HJ. Approval of final manuscript: all authors.

\section{Conflicts of Interest}

No potential conflict of interest relevant to this article was reported.

\section{Acknowledgments}

We sincerely thank Sun Bok Kang for her dedication and great title illustration. The manuscript was endorsed by Korean College of Rheumatology (KCR), Korean Heart Rhythm Society (KHRS), Korean Pediatric Heart Society (KPHS), Korean Society of Heart Failure (KSHF), Korean Society of Cardiovascular Imaging (KOSCI), Korean Society of Echocardiography (KSE), Korean Society of Interventional Cardiology (KSIC), Korean Society for Transplantation (KST), Korean Society for Laboratory Medicine (KSLM), Korean Pulmonary Hyperten- sion Society in Korean Society of Hypertension (KPHS/KSH), and Korean Association of Clinical Cardiology (KACC).

\section{Funding}

No funding to declare.

\section{References}

1. Galie N, Humbert M, Vachiery JL, Gibbs S, Lang I, Torbicki A, et al. $2015 \mathrm{ESC} / \mathrm{ERS}$ Guidelines for the diagnosis and treatment of pulmonary hypertension: The Joint Task Force for the Diagnosis and Treatment of Pulmonary Hypertension of the European Society of Cardiology (ESC) and the European Respiratory Society (ERS): endorsed by: Association for European Paediatric and Congenital Cardiology (AEPC), International Society for Heart and Lung Transplantation (ISHLT). Eur Heart J 2016;37:67-119.

2. Fukuda K, Date H, Doi S, Fukumoto Y, Fukushima N, Hatano $\mathrm{M}$, et al. Guidelines for the Treatment of Pulmonary Hypertension (JCS 2017/JPCPHS 2017). Circ J 2019;83:842-945.

3. Kovacs G, Berghold A, Scheidl S, Olschewski H. Pulmonary arterial pressure during rest and exercise in healthy subjects: a systematic review. Eur Respir J 2009;34:888-94.

4. Hoeper MM, Bogaard HJ, Condliffe R, Frantz R, Khanna D, Kurzyna M, et al. Definitions and diagnosis of pulmonary hypertension. J Am Coll Cardiol 2013;62:D42-50.

5. Galie N, McLaughlin VV, Rubin LJ, Simonneau G. An overview of the 6th World Symposium on Pulmonary Hypertension. Eur Respir J 2019;53:1802148.

6. Hoeper MM, Lee SH, Voswinckel R, Palazzini M, Jais X, Marinelli A, et al. Complications of right heart catheterization procedures in patients with pulmonary hypertension in experienced centers. J Am Coll Cardiol 2006;48:2546-52.

7. Rich S, Kaufmann E, Levy PS. The effect of high doses of calcium-channel blockers on survival in primary pulmonary hypertension. N Engl J Med 1992;327:76-81.

8. Sitbon O, Humbert M, Jais X, Ioos V, Hamid AM, Provencher $S$, et al. Long-term response to calcium channel blockers in idiopathic pulmonary arterial hypertension. Circulation 2005;111:3105-11.

9. Simonneau G, Montani D, Celermajer DS, Denton CP, Gatzoulis MA, Krowka M, et al. Haemodynamic definitions and updated clinical classification of pulmonary hypertension. Eur Respir J 2019;53:1801913.

10. Simonneau G, Gatzoulis MA, Adatia I, Celermajer D, Denton C, Ghofrani A, et al. Updated clinical classification of pulmonary hypertension. J Am Coll Cardiol 2013;62:D34-41.

11. Peacock AJ, Murphy NF, McMurray JJ, Caballero L, Stewart S. An epidemiological study of pulmonary arterial hypertension. Eur Respir J 2007;30:104-9. 
12. Song S, Lee SE, Oh SK, Jeon SA, Sung JM, Park JH, et al. Demographics, treatment trends, and survival rate in incident pulmonary artery hypertension in Korea: a nationwide study based on the health insurance review and assessment service database. PLoS One 2018;13:e0209148.

13. Chung WJ, Park YB, Jeon CH, Jung JW, Ko KP, Choi SJ, et al. Baseline characteristics of the Korean Registry of Pulmonary Arterial Hypertension. J Korean Med Sci 2015;30:1429-38.

14. Simonneau G, Robbins IM, Beghetti M, Channick RN, Delcroix M, Denton CP, et al. Updated clinical classification of pulmonary hypertension. J Am Coll Cardiol 2009;54(1 Suppl):S43-S54.

15. Soubrier F, Chung WK, Machado R, Grunig E, Aldred M, Geraci M, et al. Genetics and genomics of pulmonary arterial hypertension. J Am Coll Cardiol 2013;62:D13-21.

16. Jang AY, Kim BG, Kwon S, Seo J, Kim HK, Chang HJ, et al. Prevalence and clinical features of bone morphogenetic protein receptor type 2 mutation in Korean idiopathic pulmonary arterial hypertension patients: the PILGRIM explorative cohort. PLoS One 2020;15:e238698.

17. Eyries M, Montani D, Girerd B, Perret C, Leroy A, Lonjou C, et al. EIF2AK4 mutations cause pulmonary veno-occlusive disease, a recessive form of pulmonary hypertension. Nat Genet 2014;46:65-9.
18. Klinger JR, Elliott CG, Levine DJ, Bossone E, Duvall L, Fagan K, et al. Therapy for pulmonary arterial hypertension in adults: update of the CHEST guideline and expert panel report. Chest 2019;155:565-86.

19. Lang RM, Badano LP, Mor-Avi V, Afilalo J, Armstrong A, Ernande $\mathrm{L}$, et al. Recommendations for cardiac chamber quantification by echocardiography in adults: an update from the American Society of Echocardiography and the European Association of Cardiovascular Imaging. Eur Heart J Cardiovasc Imaging 2015;16:233-70

20. Taichman DB, McGoon MD, Harhay MO, Archer-Chicko C, Sager JS, Murugappan M, et al. Wide variation in clinicians' assessment of New York Heart Association/World Health Organization functional class in patients with pulmonary arterial hypertension. Mayo Clin Proc 2009;84:586-92.

21. Sun XG, Hansen JE, Oudiz RJ, Wasserman K. Exercise pathophysiology in patients with primary pulmonary hypertension. Circulation 2001;104:429-35.

22. Warwick G, Thomas PS, Yates DH. Biomarkers in pulmonary hypertension. Eur Respir J 2008;32:503-12.

23. Thomas CA, Anderson RJ, Condon DF, de Jesus Perez VA. Diagnosis and management of pulmonary hypertension in the modern era: insights from the 6th World Symposium. Pulm Ther 2020;6:9-22. 